\title{
HIF-1 expression is associated with CCL2 chemokine expression in airway inflammatory cells: implications in allergic airway inflammation
}

Guillermina J Baay-Guzman ${ }^{1,2}$, Ilona G Bebenek ${ }^{3,4}$, Michelle Zeidler ${ }^{5,11}$, Rogelio Hernandez-Pando ${ }^{6}$, Mario I Vega ${ }^{7}$, Eduardo A Garcia-Zepeda ${ }^{8}$, Gabriela Antonio-Andres ${ }^{1}$, Benjamin Bonavida ${ }^{9,10}$, Marc Riedl ${ }^{5}$, Eric Kleerup ${ }^{11}$,

Donald P Tashkin ${ }^{11}$, Oliver Hankinson ${ }^{3}$ and Sara Huerta-Yepez ${ }^{1 *}$

\begin{abstract}
Background: The pathogenesis of allergic airway inflammation in asthmatic patients is complex and characterized by cellular infiltrates and activity of many cytokines and chemokines. Both the transcription factor hypoxia inducible factor-1 (HIF-1) and chemokine CCL2 have been shown to play pivotal roles in allergic airway inflammation. The interrelationship between these two factors is not known. We hypothesized that the expression of HIF-1 and CCL2 may be correlated and that the expression of CCL2 may be under the regulation of HIF-1. Several lines of evidence are presented to support this hypothesis.
\end{abstract}

Methods: The effects of treating wild-type OVA (ovalbumin)-sensitized/challenged mice with ethyl-3, 4-dihydroxybenzoate (EDHB), which upregulate HIF, on CCL2 expression, were determined. Mice conditionally knocked out for HIF-1 $\beta$ was examined for their ability to mount an allergic inflammatory response and CCL2 expression in the lung after intratracheal exposure to ovalbumin. The association of HIF-1a and CCL2 levels was also measured in endobronchial biopsies and bronchial fluid of asthma patients after challenge.

Results: We show that both HIF-1a and CCL2 were upregulated during an OVA (ovalbumin)-induced allergic response in mice. The levels of HIF-1a and CCL2 were significantly increased following treatment with a pharmacological agent which upregulates HIF-1a, ethyl-3,4-dihydroxybenzoate (EDHB). In contrast, the expression levels of HIF-1a and CCL2 were decreased in the lungs of mice that have been conditionally knocked out for ARNT (HIF-1ß) following sensitization with OVA when compared to levels in wild type mice. In asthma patients, the levels of HIF-1a and CCL2 increased after challenge with the allergen.

Conclusions: These data suggest that CCL2 expression is regulated, in part, by HIF-1 in the lung. These findings also demonstrate that both CCL2 and HIF-1 are implicated in the pathogenesis of allergic airway inflammation.

Keywords: Allergic airway inflammation, Asthma, Hypoxia inducible factor-1, CCL2, Arylhydrocarbon receptor nuclear translocator

\footnotetext{
* Correspondence: shuertay@gmail.com

${ }^{1}$ Hospital Infantil de Mexico, Federico Gomez, Unidad de Investigacion en Enfermedades Oncologicas, Dr. Marquez No 262, Col. Doctores, Delegacion Cuahutemos, Mexico City, Mexico CP. 06720

Full list of author information is available at the end of the article
} 


\section{Background}

Asthma is a global health concern with increasing prevalence of morbidity and mortality worldwide [1]. Asthma is a chronic inflammatory disease resulting in the overproduction of mucus, airflow obstruction, and airway hyperresponsiveness. The pathogenesis of asthma includes an exaggerated immune response to an allergen. This, in turn, activates CD4 T helper-2 (Th2) lymphocytes and the release of specific cytokines (including IL-4, IL-5, and IL-13) and chemokines (including CCL2, CCL3, CCL4, CCL5 CCL11 and CCL24) [2,3]. These cytokines and chemokines induce increased airway inflammation, including eosinophil recruitment [4].

Monocyte chemotactic protein 1 (MCP-1/CCL2) is a member of the CC family of chemokines [5], a potent chemoattractant for macrophages [6], and also attracts CD4+ and CD8+ T-lymphocytes [7]. Increasing evidence suggests that CCL2 and its haematopoietic cell receptor CC chemokine receptor 2 (CCR2) are involved in inflammatory disorders of the lungs [7]. CCL2 may have a significant role in the pathogenesis of asthma because of its ability to recruit eosinophils and monocytes, activate basophils and mast cells and induce the release of leukotriene $\mathrm{C} 4$ into the airway, all of which contribute to airway hyperresponsiveness [8]. CCL2 can also direct undifferentiated T-lymphocytes towards interleukin-4 (IL-4)-producing Th2 cells $[9,10]$. Overexpression of CCL2 has been observed in the bronchial epithelium of asthmatic patients [11]. CCL2 is also significantly upregulated after challenge in asthmatic patients [12]. Sensitization and challenge with cockroach $\mathrm{Ag}$ in mice, in which CCL2 activity was blocked with a specific antimurine MCP-1 antibody, showed a decrease in several allergic inflammatory manifestations such as histamine and LTC4 levels, and attenuated airway hyperreactivity in sensitized mice [13]. Additionally, CCR2-/- mice have an attenuated airway hyperreactive response during allergen challenge or after direct instillation of CCL2, implicating a CCR2-mediated mechanism. Furthermore the neutralization of CCL2 during the allergic airway response decreased histamine in the BAL. [8]. These results support an important role for CCL2 in the asthmatic response.

CCL2 is known to be regulated by oxidative stress, cytokines and growth factors. In addition, CCL2 is also associated with hypoxic regulation. CCL2 was induced by both hypoxia and $\mathrm{CoCl}_{2}$ in human astrocytes and the promoter of CCL2 contains hypoxia response elements (HREs) which can bind hypoxia inducible factor1 (HIF-1) [14].

The transcriptional response to hypoxia is primarily mediated by the hypoxia inducible factor (HIF) family of transcription factors. HIFs are heterodimeric proteins containing one $\alpha$ subunit and one $\beta$ subunit. HIF- $1 \alpha$ and HIF- $2 \alpha$ (collectively called the HIF- $\alpha$ subunits) are both expressed widely, as is HIF-1 $\beta$ (also called the Aryl Hydrocarbon Receptor Nuclear Translocator [ARNT]). All of the HIF subunits belong to the basic helix-loophelix (bHLH)/Per-ARNT-Sim (PAS) domain family of transcription factors. The $\beta$ subunit is constitutively expressed, and the regulation of HIFs occurs mainly via effects on the $\alpha$ subunits. Under normoxic conditions, the HIF- $\alpha$ subunits are hydroxylated on key proline residues, which allows for their recognition by the von Hippel-Lindau (pVHL) tumor suppressor protein, the substrate recognition component of an E3 ubiquitin ligase complex that targets HIF- $\alpha$ for proteasomal degradation. Hydroxylation of both prolines is catalyzed by a family of three prolyl hydroxylases. Another level of HIF- $1 \alpha$ regulation occurs through the hydroxylation of an asparagine residue by Factor Inhibiting Hypoxia Inducible Factor- $1 \alpha$ (FIH) which prevents HIF- $1 \alpha$ from interacting with the coactivator p300 under normoxic conditions $[15,16]$. During hypoxia, prolyl hydroxylases are inactive allowing HIF- $1 \alpha$ to dimerize and form a complex with its partner ARNT and then binds HREs in the promoter regions of target genes, upregulating their transcription [17].

HIF- $1 \alpha$ can be upregulated during inflammation as a consequence of the hypoxic microenvironment or via a hypoxia-independent mechanism [18]. HIF-1 $\alpha$ can also regulate the expression of several relevant factors, including pro-inflammatory cytokines, chemokines, and adhesion molecules. Enhanced levels of HIF- $1 \alpha, 2 \alpha$ and VEGF have been identified in lung tissues and bronchial epithelial cells of asthmatic patients as well as in the bronchial lavage of asthmatic patients, where they were associated with elevated levels of eosinophil counts as compared to persons without asthma [19]. Moreover, a direct correlation was found between the levels of HIF$1 \alpha, 2 \alpha$ and VEGF [19].

We recently reported that HIF- $1 \alpha$ is increased after allergic challenge in asthma and rhinitis patients $[20,21]$. However, the relationship between HIF-1 and CCL2 in allergic airway inflammation has not yet been described. In this study we found a direct association between HIF- $1 \alpha$ and CCL2 expression in allergic airway inflammation in an animal model. CCL2 was upregulated in vivo after treatment with a HIF- $1 \alpha$ inducer. In contrast, CCL2 was downregulated in mice deficient in ARNT, an obligatory subunit necessary for HIF-1 activity. In addition, we also found a direct correlation between upregulated levels of CCL2 and HIF-1 after challenge in samples from asthmatic patients. These findings demonstrate for the first time, a role for HIF$1 \alpha$ in the regulation of CCL2 expression in airway inflammatory disease. 


\section{Methods}

\section{Breeding and genotyping of mice}

The original Arnt ${ }^{\mathrm{F}}$ allele contained a neo cassette, which was excised as described previously [22]. The $\mathrm{Arnt}^{\mathrm{F} / \mathrm{F}}$ mice, which were of a mixed C57BL/6, 129/Sv and FVB/ $\mathrm{N}$ genetic background, were back crossed to homozygous Mx1-Cre + mice in a C57BL/6 genetic background (Jackson Laboratory, Bar Harbor, Maine). Progeny from this cross were then backcrossed at least ten successive times to generate a mouse strain in a $100 \% \mathrm{C} 57 \mathrm{BL} / 6$ background. Genotyping of the $\operatorname{Arnt}^{\mathrm{F}}$ and $\mathrm{Arnt}^{\Delta}$ alleles was performed by PCR as described previously [23]. The Mx1-Cre transgene was genotyped with PCR primers directed at the Cre gene as described previously [23]. Mx1-Cre+/- heterozygotes could not be distinguished from $\mathrm{Mx} 1-\mathrm{Cre}+/+$ homozygotes by this procedure, and these genotypes are collectively referred to as Mx1-Cre+. $\mathrm{KO}$ mice were obtained and maintained in the facilities of the University of California, Los Angeles (UCLA) (USA).

$\mathrm{Balb} / \mathrm{c}$ male mice between 6 to 8 weeks old were obtained and maintained in the facilities of the Instituto Nacional de Ciencias Medicas y de la Nutrición "Salvador Zubirán” (INCMN) (Mexico City). Mice were maintained in a pathogen-free environment, in a temperaturecontrolled room with 12-h dark/light cycles, and allowed food and water ad libitum. All experiments were performed in accordance with UCLA and INCMN animal regulations.

\section{Treatment of mice}

Induction of the Cre protein and,thus, deletion of the Arnt allele in Mx1-Cre:Arnt ${ }^{\mathrm{F} / \mathrm{F}}$ mice was induced by intraperitoneal (i.p) injection with $500 \mu \mathrm{g}$ of polyinosinic-polycitidylic acid (pIpC, Sigma, St.Louis, $\mathrm{MO}$ ) in PBS on three consecutive times two days apart. Sensitization was elicited by two i.p. treatments, five days apart, with $100 \mu \mathrm{g}$ of chicken egg ovalbumin (OVA, grade V, Sigma, St. Louis, MO) emulsified in $1 \mathrm{mg}$ of aluminum hydroxide (Pierce Chemical, USA) in a total volume of $100 \mu \mathrm{L}$. Mice were challenged via intratracheal (i.t.) administration of $0.75 \%$ OVA on two or three occasions as indicated. Control mice received vehicle solvent only.

Where indicated, mice were i.p. injected with $100 \mathrm{mg} / \mathrm{kg}$ of ethyl 3,4, dihydroxy benzoate (EDHB) (Sigma, St.Louis, $\mathrm{MO}$ ) in $10 \%$ DMSO. In all cases treatment with the $10 \%$ DMSO vehicle was used as negative control. Mice were euthanized by inhalation of isofluorane. All experiments were performed in accordance with UCLA and INCMN regulations.

\section{Lung histology and morphometric analysis}

After the mice were euthanized by exsanguination, the lungs were filled intratracheally with a fixative (absolute ethanol). The lungs were then removed and fixed with ethanol, dehydrated and embedded in paraffin. For histological examination, 4- $\mu \mathrm{m}$ sections of fixed embedded tissues were cut on a Seea Meins model rotary microtome (Germany), placed on glass slides, and deparaffinized. The slides were stained sequentially with hematoxylin and eosin (H\&E) to assess inflammatory cell infiltration. The area $\left(\mu \mathrm{m}^{2}\right)$ of inflammation was calculated from venules with diameters of $100-200 \mu \mathrm{m}$ and from bronchioli $150-300 \mu \mathrm{m}$ in diameter. Four blood vessels or bronchioli from each of four to six lung sections were analyzed for each experimental group, and results are expressed as the average and standard deviations of the infiltrated area. Mucus production was assessed in lung sections stained with periodic acid Schiff (PAS), focusing on bronchioli of $150-300 \mu \mathrm{m}$ in diameter. The PAS-positive material was measured in $200 \mu \mathrm{m}$ squares $\left(40,000 \mu \mathrm{m}^{2}\right)$. Five bronchioli from four to six lung sections were analyzed for each experimental group, and the results are expressed as the average and standard deviation of PAS-positive area $(\mu \mathrm{m})$. Slides were analyzed under an Olympus BX-40 microscope. Quantification analysis was performed using Image Proplus 6.2 software (Media Cybernetics, Bethesda, MD).

\section{Immunohistochemistry}

Deparaffinized 4- $\mu \mathrm{m}$ sections were used for immunohistochemical analysis of HIF- $1 \alpha$ and CCL2. Briefly, antigen retrieval was performed by immersing the slides in $0.01 \mathrm{M}$ sodium citrate, $\mathrm{pH}$ 6.0, for twentyfive minutes in boiling water. Endogenous peroxidase activity was inhibited by immersing the slides in $3 \% \mathrm{H}_{2} \mathrm{O}_{2}$-methanol for $20 \mathrm{~min}$, and background nonspecific binding was reduced by incubating with $1 \%$ normal swine serum (NSS) in PBS for $60 \mathrm{~min}$. The slides were incubated overnight at room temperature with antibodies against HIF-1 $\alpha$ (1:250 dilution, Santa Cruz Biotechnology, Santa Cruz, CA), and CCL2 (1:500 dilution, Santa Cruz). Finally, the slides were washed five times in PBS $1 \mathrm{X}, \mathrm{pH}$ 7.4, for eight minutes. In order to reduce variability, all samples from each group were processed at the same time in a single experiment using a single batch of antibody diluted in PBS with normal swine serum. After washing, the tissues were incubated with a biotinylated secondary antibody anti-rabbit IgG (1:500) (Santa Cruz) for HIF-1 $\alpha$ or donkey anti-goat IgG biotin conjugate (1:500 Santa Cruz) for CCL2 for 30 minutes at room temperature followed by incubation with a streptavidinHRP for 30 minutes at room temperature, and revealed with 3,3'-diaminobenzidine tetra-hydrochloride (DAKO, Carpinteria, CA, USA). The reaction was arrested with water, and the slides were counterstained with hematoxylin. Thereafter, the tissues were washed in distilled water for five minutes, dehydrated sequentially in 
70\%, 90\% and 100\% ethanol, ethanol/xylene and xylene, and then mounted with Cytoseal-60 (Fisher Scientific, Pittsburgh, PA). Finally, the slides were analyzed under light microscopy (Olympus BX-40).

\section{Double-immunofluorescence}

HIF- $1 \alpha$ and CCL2 colocalization in cells from BAL fluid from asthmatic patients was confirmed using immunofluorescence. Cells from BAL fluid from asthmatic patients post challenge were fixed with $4 \%$ paraformaldehyde with $0.2 \%$ Triton X-100. After blocking (5\% goat serum, $3 \% \mathrm{BSA}$, and $0.2 \%$ Triton X-100 in PBS), the cells were incubated with anti-HIF-1 $\alpha$ antibody for $1 \mathrm{~h}$ at $4^{\circ}$ C. After incubation, the cells were treated with an FITCconjugated anti-rabbit antibody (Jackson Immunoresearch, PA). For CCL2 detection, the cells were treated with murine anti-CCL2 antibody and developed with phycoeritryn-conjugated anti-goat antibody (Jackson Immunoresearch, PA). After double-staining, the cells were mounted using Gel/Mount (Biomeda Corp.). Immunofluorescence was examined and photographed with a Olympus B24 microscope equipped with epifluorescence illumination.

\section{Bronchoalveolar lavage (BAL) of mice}

After the mice were euthanized by exsanguination, the tracheas were exposed and intubated with a polyethylene catheter. The BAL fluid was collected by washing with two separate aliquots of $1 \mathrm{ml}$ of sterile saline (SS) containing $2 \%$ of fetal bovine serum (FBS) through the trachea. The cells from both washes were harvested by centrifugation $\left(500 \times \mathrm{g}\right.$ for $10 \mathrm{~min}$ at $\left.4{ }^{\circ} \mathrm{C}\right)$. CCL2 in BAL supernatant fluid was determined by ELISA.

\section{ELISA}

The quantification of serum levels of CCL2 was performed using an ELISA protocol. Briefly, micro ELISA plates were coated with monoclonal CCL2 antibody (R\&D systems, Minneapolis, MN) diluted 1:500, for 2 hours. The plates were washed three times, and then blocked with chicken egg OVA (Sigma, St.Louis, MO). The plates were then washed three times and secondary antibody ( $R \& D$ systems, Minneapolis, MN), diluted at 1:1000 was added. After four hours, AP-strepavidin (Zymed, San Francisco, CA) was added at a dilution of 1:1000, and the plate was incubated for 1 hour. BluePhos (KPL, Gaithersburg, MD) was used to develop the reaction and the plate was read at $650 \mathrm{~nm}$ within 2 hours.

\section{Human subjects}

Nine mild to moderate asthmatic subjects who were steroid naïve and cat allergic were studied ( 6 female $/ 3$ male; age $26 \pm 7.0$ years; mean $\mathrm{FEV}_{1}$ [forced expiratory volume in 1 second] of $96 \pm 8 \%$ ). Subjects underwent a baseline fiberoptic bronchoscopy (FOB) in which endobronchial biopsies and bronchoalveolar lavage (BAL) were obtained. Three days later, subjects underwent a naturalistic cat room challenge (previously described) [24,25] until their $\mathrm{FEV}_{1}$ dropped by $20 \%$ or for an hour, whichever occurred first (Fel d 1 cat antigen protein concentration was 17.2 $\pm 5.8 \mathrm{ng}$ Fel d 1/cu meter air). Three days later, subjects again underwent FOB to obtain biopsies and BAL. Two endobronchial biopsies from a randomly selected side were obtained from subsegmental or segmental carinii and placed in cassettes in $4 \%$ paraformaldehyde, transferred to $70 \% \mathrm{ETOH}$ and then paraffin embedded and stored until immunohistochemistry (IHC) was performed. Bronchoalveolar lavage was performed with normal saline in (sub) segments of the right middle lobe or lingula in a randomized manner. 40 and $50 \mathrm{ml}$ were instilled and withdrawn by syringe aspiration, filtered through a $100 \mu$ filter, and placed on ice. The BAL was spun down at 3000 RPM for 10 minutes, the cell pellet was resuspended in fetal bovine serum (FBS), and cytospins were made $(100,000$ cells per slide) and stained for cell count and differential (H\&E) or fixed in $4 \%$ paraformaldehyde and stored in PBS for IHC.

All subjects signed an informed consent and study activities were approved by the Human Subject Protection Committee of the University of California, Los Angeles.

\section{Cytoimmunostaining}

Cytospins from BAL were fixed in $10 \%$ formalin and stained for HIF-1 $\boldsymbol{\alpha}$ and CCL2 as described above in the immunohistochemistry protocol.

\section{Statistical analysis}

Data are expressed as mean \pm S.E.M. Statistical comparisons were performed using one-way analysis of variance followed by ANOVA. Significant differences between groups were determined using the unpaired Student's t-test. Statistical significance was set at $\mathrm{p}<0.05$. For the correlation between HIF-1 $\boldsymbol{\alpha}$ and CCL2 expression in the human samples, we used Pearson's correlation analysis.

\section{Results}

\section{Induction of HIF-1a results in CCL2 overexpression}

We investigated whether upregulation of HIF-1 $\boldsymbol{\alpha}$ would affect CCL2 expression. We used EDHB, which inhibits prolyl hydroxylases competitively with regard to two of their cosubstrates, oxoglutarate and ascorbate [26]. Balb/c mice were sensitized with OVA, i.p., on days 0 and 5. Next, EDHB was injected, i.p. on three occasions, one day before each ntratracheal challenge with OVA, and the mice were sacrificed four days after the last OVA treatment (Figure 1A). The inflammatory response was measured in lung tissue using H\&E and PAS staining (Figure 1B). OVA treatment led to an increase in perivascular (Figure 1Bc) 
and peibronchiolar (Figure 1Bg) inflammation. Treatment with OVA and EDHB (Figure 1Bd and 1Bh) led to a markedly enhanced inflammatory response compared with treatment with OVA alone. EDHB elicited a modest inflammatory response by itself (Figure $1 \mathrm{Bb}$ and $1 \mathrm{Bf}$ ). In addition, PAS staining showed an increase in mucus production in mice treated with OVA (Figure 1Bk) as compared to saline treated mice (Figure 1Bi). Enhanced mucus production was observed after treatment with the combination of OVA and EDHB (Figure 1Bl) as compared to OVA alone. EDHB treatment alone did not induce mucus production (Figure 1Bj). Quantitation of the

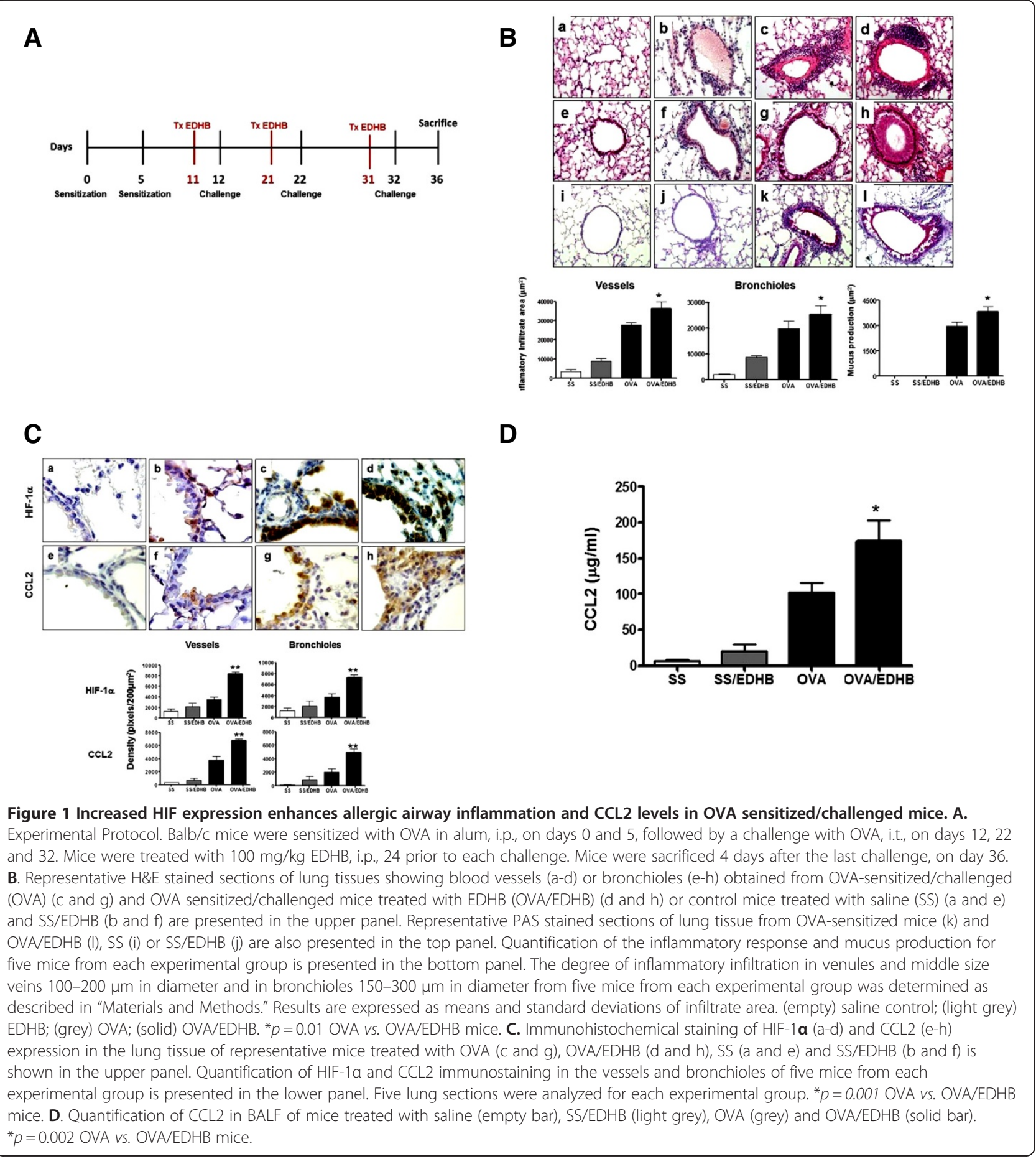


inflammatory response (Figure 1B bottom panel) shows that the observed differences were statistically significant.

Immunohistochemical analysis (Figure 1C) demonstrated that OVA and EDHB enhanced the expression of HIF-1 $\alpha$ (Figure $1 \mathrm{Cd}$ ) and CCL2 (Figure $1 \mathrm{Ch}$ ) above the levels elicited by OVA alone (Figure $1 \mathrm{Cc}$ and g). VEGF expression was also significantly upregulated (data not shown) [21]. Lungs of saline-treated mice did not show significant expression of either HIF-1 $\alpha$ or CCL2 (Figure $1 \mathrm{Ca}$ and e). EDHB alone slightly upregulated HIF- $1 \alpha$ and CCL2 expression (Figure $1 \mathrm{Cb}$ and $1 \mathrm{Cf}$ ). Quantification of the expression of HIF- $1 \alpha$ and CCL2 revealed that the enhanced expression of both proteins in response to OVA and OVA and EDHB treatment was statistically significant (Figure $1 \mathrm{C}$, bottom panel). Furthermore, using flow cytometry, we determined that macrophages were the principal cells expressing HIF- $1 \alpha$ and CCL2, while B and T cells expressed lower levels of these proteins; dendritic cells expressed modest amounts of the proteins. In addition we found a higher amount of HIF-1 and CCL2 doubly-stained positive cells in OVAsensitized mice as compared with control mice (data not shown).

Thus, during airway inflammation, CCL2 was upregulated by EDHB, an agent which upregulates HIF and at least one of its target genes, VEGF (data not shown) [21]. In addition, we analyzed the expression of CCL2 in the lavage of treated mice. We found a significant increase in the expression of CCL2 in the lavage fluid of the OVA and EDHB treated mice as compared to mice treated with OVA alone (Figure 1D).

\section{CCL2 expression is reduced in mice deficient in ARNT}

Mice that are homozygous for an Arnt null allele (i.e. Arnt knockout mice) die in utero $[27,28]$. We, therefore, used conditionally knocked out mice in which deletion of the Arnt gene can be deleted in adulthood [21]. Briefly, the ARNT allele in the $\mathrm{Arnt}^{\mathrm{F} / \mathrm{F}}: \mathrm{Mx} 1-\mathrm{Cre}$ mice can be deleted with treatment with polyinosinicpolycytidylic acid (pIpC), which activates transcription of the Cre gene. The Cre recombinase catalyzes deletion of the genomic segment between two loxP sites. Thus, in $\mathrm{Arnt}^{\mathrm{F} / \mathrm{F}}: \mathrm{Mx} 1-\mathrm{Cre}^{+}$mice i.p. injected with $\mathrm{pIpC}$, inactivation of the Arnt gene occurs in most tissues of the body and at about $80 \%$ efficiency in the lung [22]. In order to test our hypothesis that HIF-1 plays a role in CCL2 expression during airway inflammation, we examined the expression of CCL2 in mice sensitized and challenged with OVA and deficient for the ARNT allele.

Eight $\operatorname{Arnt}^{\mathrm{F} / \mathrm{F}}: \mathrm{Mx} 1-\mathrm{Cre}$ mice per group were used in these experiments. The mice received $\mathrm{pIpC}$ to delete the Arnt gene. Mice were subjected to a previously described allergenic protocol [20], involving two intraperitoneal treatments with the allergen OVA, and the adjuvant, alum, followed by three intratracheal administrations of OVA (Figure 2A). Routine H\&E stained lung sections were used to evaluate the degree of inflammatory cell infiltration (Figure 2B). OVA treatment in the $\mathrm{Cre}^{-}$mice elicited a marked perivascular (Figure $2 \mathrm{Bb}$ ) and peribronchiolar (Figure 2Be) inflammatory infiltration, comprised mostly of mononuclear cells. The $\mathrm{Cre}^{+}$mice treated with OVA exhibited a marked reduction in the degree of perivascular (Figure 2Bc) and peribronchiolar (Figure 2Bf) inflammatory cell infiltration, as compared to the similarly treated $\mathrm{Cre}^{-}$mice (Figure $2 \mathrm{Bb}$ and e). Saline treated mice did not show any significant inflammatory response (Figure $2 \mathrm{Ba}$ and $\mathrm{d}$ ). The decrease in infiltration in the $\mathrm{Cre}^{+}$mouse lungs was statistically significant (Figure 2B, bottom panel).

We also analyzed the expression of HIF- $1 \alpha$ and CCL2 in the lungs of these mice. Representative lung sections from each experimental group and quantitative analysis of the staining in all the mice are presented in Figure 2C. The expression of HIF- $1 \alpha$ and CCL2 was drastically reduced in the $\mathrm{Cre}^{+} / \mathrm{OVA}$ treated mice (Figure 2Cc and f), as compared to the Cre-/OVA mice (Figure $2 \mathrm{Cb}$ and e). OVA treatment of the $\mathrm{Cre}^{-}$mice (Figure $2 \mathrm{Cb}$ and e) increased HIF-1 $\alpha$ levels in the nucleus and CCL2 levels in the cytoplasm of inflammatory and bronchiolar epithelial cells as compared to untreated mice (Figure 2Ca and d). Reduction of HIF-1 $\alpha$ and CCL2 in both vessels and bronchioles in $\mathrm{Cre}^{+}$/OVA mice as compared to Cre-/OVA mice was statistically significant (Figure 2C bottom panel).

\section{CCL2 expression correlates with increased HIF-1a} expression in asthmatic patients after allergen challenge We also evaluated CCL2 expression in human samples $(n=9)$ in order to validate our findings in mice. The subjects general clinical characteristics are described in Table 1. We examined HIF- $1 \alpha$ and CCL2 expression in bronchial lavage fluid (Figure 3A) and lung tissue (Figure 3B) derived from asthmatic patients before and after allergen challenge. Figure $3 \mathrm{~A}$ shows representative photomicrographs of HIF-1 $\alpha$ (Figure $3 \mathrm{Aa}$ and $\mathrm{b}$ ) and CCL2 (Figure 3Ac and d) immunostained cells obtained from lavage in asthmatic patients before (Figure 3Aa and c) and after (Figure $3 \mathrm{Ab}$ and d) exposure to antigen challenge. HIF- $1 \alpha$ and CCL2 expression was significantly increased in cells from lavage after allergen challenge (Figure 3A, right panel). In order to demonstrate the coexpression of HIF-1 $\boldsymbol{\alpha}$ and CCL2 in cells from lavage, we performed double-immunostaining (Figure 3B). Our results demonstrate that HIF-1 $\boldsymbol{\alpha}(3 \mathrm{Ba})$ and CCL2 $(3 \mathrm{Bb})$ were colocalized in the same cells (Figure 3Bc).

In Figure $3 \mathrm{C}$ we show HIF-1 $\boldsymbol{\alpha}$ (Figure $3 \mathrm{Ca}$ and $\mathrm{b}$ ) and CCL2 (Figure 3Cc and d) immunostaining of lung tissue 


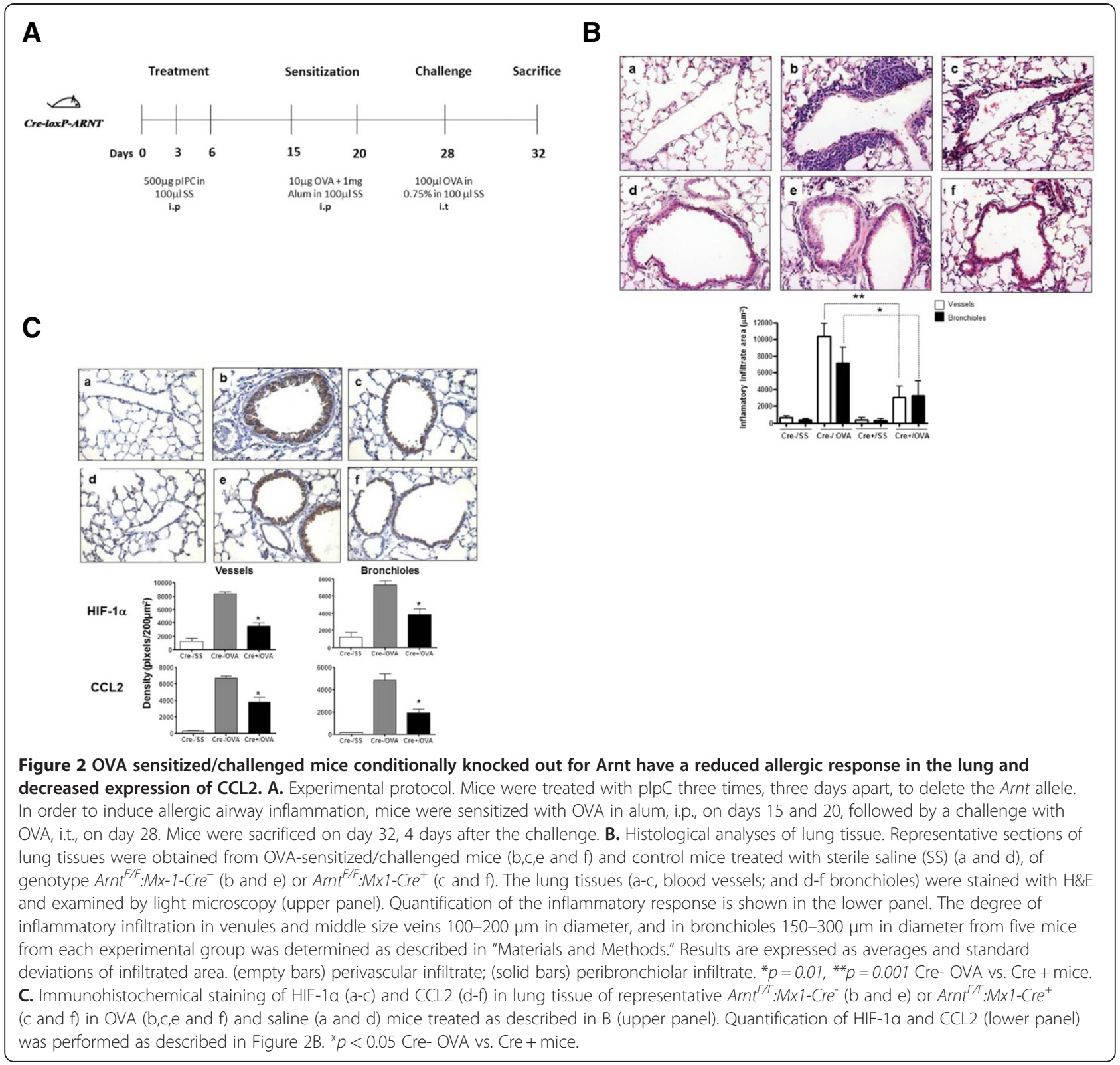

from asthmatic patients, before (Figure $3 \mathrm{Ca}$ and $\mathrm{c}$ ) and after (Figure $3 \mathrm{Cb}$ and $\mathrm{d}$ ) exposure to challenge. Expression of HIF-1 $\boldsymbol{\alpha}$ was predominantly nuclear, although some cytoplasmic staining was observed. Expression of CCL2 was predominantly cytoplasmic or in the membrane. The right panel of Figure $3 \mathrm{C}$ shows quantification of the density of the expression of HIF-1 $\alpha$ and CCL2 in the lung tissue. Expression of HIF-1 $\boldsymbol{\alpha}$ and CCL2 was significantly higher after challenge. Furthermore, there was a direct correlation between HIF-1 $\boldsymbol{\alpha}$ and CCL2 expression after challenge, as measured by inmunostaining (Figure 3D), ("p $\left.<0.05,{ }^{*} \mathrm{r}=0.7930\right)$.
These findings therefore build on our animal studies and suggest that HIF-1 plays an important role in the regulation of CCL2 during allergic disease in the human.

\section{Discussion}

Previous observations have implicated HIF-1 in the development of asthma [21]. Hypoxia is a potent stimulus for inflammation and remodeling, and HIF-1 can activate transcription of several inflammatory cytokines, chemokines and growth factors, including VEGF, as well as matrix remodeling proteins, such as procollagen and matrix metalloproteinases [29]. N-acetylcysteine amide 
Table 1 Clinical features of patients with asthma

\begin{tabular}{ll}
\hline Asthmatic subjects & \\
\hline Subjects $n$ & 9 \\
Age years & $26(18-43)$ \\
Sex M:F & $3: 6$ \\
FEV1\% predicted & $94.67(76-112)$ \\
FEV1 (L) & $3.9(2.9-5.0)$ \\
FEV1 at 0 min (L) [CRC] & $3.7(2.6-4.9)$ \\
FEV1 Lowest $(L)[C R C]$ & $3.39(2.5-4.3)$ \\
\% FEV1 decline $[C R C]$ & $8.72(-8.3-32.1)$ \\
\hline
\end{tabular}

(AD4), a newly developed antioxidant thiol compound, attenuated airway inflammation as well as hyperresponsiveness by regulating HIF-1 $\boldsymbol{\alpha}$ and NF-kB and reducing reactive oxygen species (ROS) levels [30]. Inhibition of PPAR decreased HIF-1 and NF-kB and induced attenuation of the airway inflammatory response [31]. VEGF and HIF- $1 \boldsymbol{\alpha}$ and HIF- $2 \boldsymbol{\alpha}$ were expressed at higher levels in asthmatic patients as compared to healthy controls [19]. Most recently, Guo et al. found a significant reduction in the number of eosinophils in BAL fluid after sensitization/ challenge with OVA in mice heterozygous for a HIF- $1 \alpha$ null allele as compared to wild-type mice [32]. However, a clear mechanism of how HIF-1 is involved in perpetuating the inflammatory response is not clear because HIF-1 regulates several chemokines and cytokines. Previous studies have demonstrated that HIF-1 $\boldsymbol{\alpha}$ regulates CCL2 at the transcriptional level [14]. We provide evidence for this notion in the present study in that at least one mechanism for HIF-1-mediated inflammation in the lung during an

\section{A}
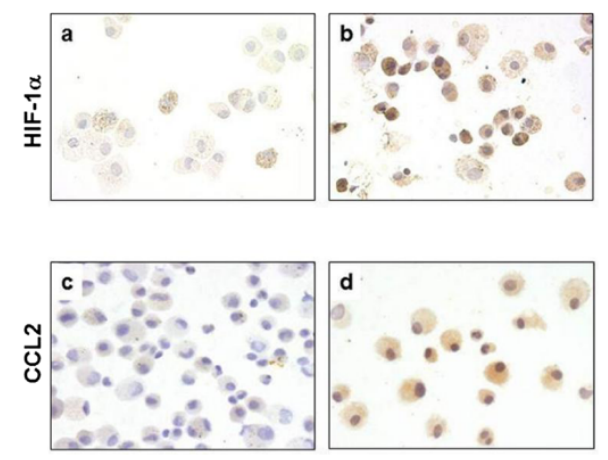

C

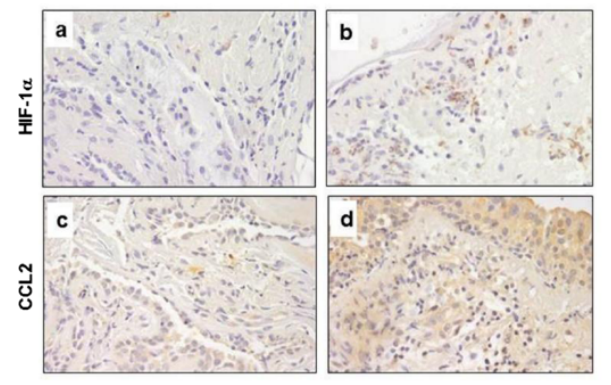

B
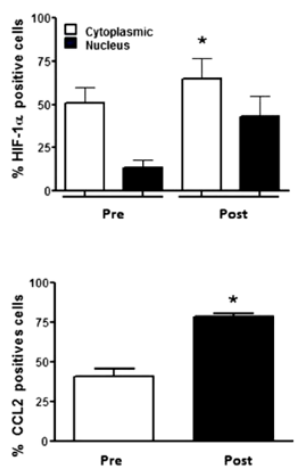

D

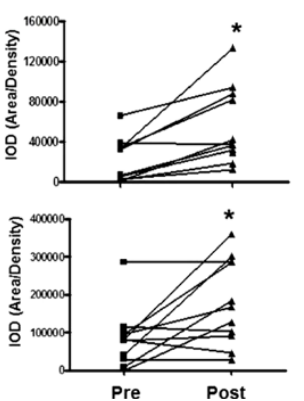

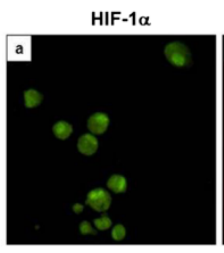
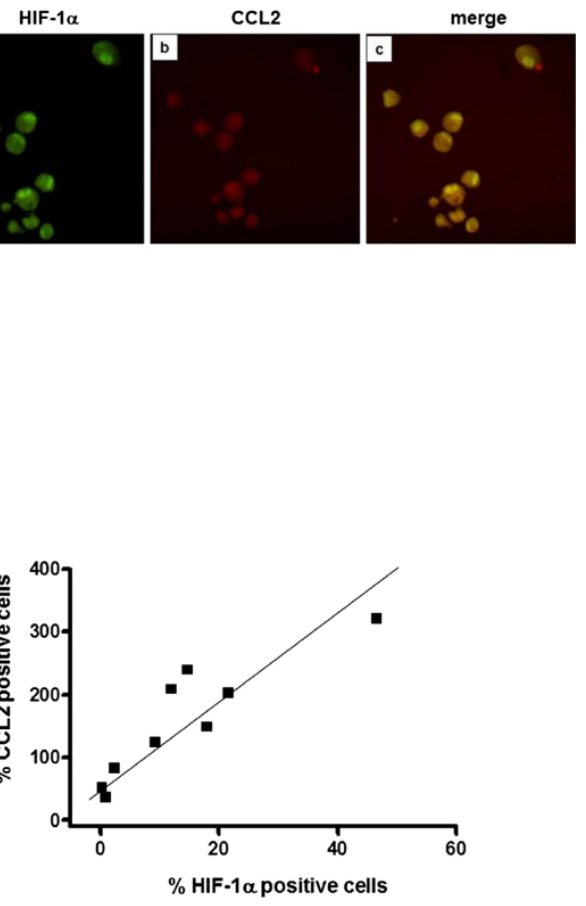

Figure 3 HIF-1a and CCL2 are both increased in BALF and lung tissue after challenge in asthmatic patients. A. Representative photomicrographs of HIF-1a ( $\mathrm{a}$ and b) and CCL2 ( $\mathrm{c}$ and d) immunostaining observed at magnification of 100X in epithelial cells from bronchial lavage before ( $a$ and $c$ ) and after ( $b$ and $d$ ) challenge. In the right panel, we quantified HIF-1 $a$ and CCL2 positive cells, before and after the challenge and found a significant increase in the expression of both genes. Student t-test ${ }^{*} p<0.05$ pre vs. post challenge HIF-1a and CCL2 expression. B. The colocalization of HIF-1 $\mathbf{a}$ and CCL2 in BAL cells from asthmatic patients was performed using double-immunofluorescence. The cells were incubated with anti-HIF-1 $\mathbf{a}$ antibody and then with FITC-conjugated anti-rabbit antibody (a). For CCL2 detection, the cells were treated with goat anti-CCL2 antibody and then treated with a secondary antibody with red fluorescence (b). The merging of fluorescence resulted in significant yellow fluorescence that indicated co-expression of HIF-1 $\mathbf{a}$ and CCL2 (c). C. Representative photomicrographs of HIF-1 $\mathbf{a}$ (a and b) and CCL2 expression (c and d) before (a and c) and after ( $b$ and $d$ ) challenge, observed at magnification of 40X in lung tissue (left panel). Both HIF-1 a and CCL2 expression was enhanced after allergen challenge in the lung tissue. In the right panel, we quantified the density of the expression of HIF-1 $\mathbf{a}$ and CCL2 before and after challenge. This analysis was performed from data collected from 9 patients. Student t-test ${ }^{*} p<0.05$ Pre vs. post challenge HIF-1a and CCL2 expression. D. Correlation analysis between HIF-1 $\mathbf{a}$ and CCL2 positively immunostained cells after challenge. Pearson's analysis $(p=0.001, r=0.878)$ post challenge HIF- $1 \mathbf{a}$ vs. CCL2. 
allergic response is via the upregulation of the proinflammatory chemokine CCL2, mainly by macrophages, which in turn, can recruit cells such as eosinophils and lymphocytes to areas of inflammation.

In a previous study conducted by our group, EDHB, a known inducer of HIF-1 $\boldsymbol{\alpha}$ both in vivo and in vitro [33], resulted in increased levels of HIF-1 $\boldsymbol{\alpha}$ and the target gene VEGF in the airway epithelium of mice [21]. EDHB also enhanced the allergenic response to OVA, including airway inflammation, goblet cell hyperplasia, and increased mucus production, indicating that HIF-1 is involved in generating the allergic inflammatory response. In order to investigate whether one of the mechanisms of HIF-1-mediated lung inflammation is via upregulation of the pro-inflammatory chemokine CCL2, we analyzed the effect of EDHB on airway inflammation in combination with OVA. Mice treated with EDHB and OVA had significantly higher expression of CCL2 than mice treated with OVA alone and these levels correlated with the expression of HIF-1 $\boldsymbol{\alpha}$ and the inflammatory response (Figure 1C). EDHB alone was sufficient to induce an inflammatory response in our model as previously observed [21] as well as CCL2 expression. To our knowledge, this is the first time that a compound known to induce HIF-1 activation has been shown to induce the expression of CCL2 in the absence of a specific allergenic stimulus.

In order to validate our hypothesis that HIF- $1 \alpha$ regulates CCL2 during allergic airway inflammation, we utilized mice conditionally knocked out for ARNT, which is an obligatory subunit of HIF-1 [34]. Arnt2 is not expressed in mouse (or human) lung [22] and, therefore, its potential effects can be excluded from our study. It should be noted that both HIF- $1 \boldsymbol{\alpha}$ and HIF- $2 \boldsymbol{\alpha}$ are expressed in the mouse lung [21], particularly in the bronchiolar epithelium and lung vascular endothelium. Therefore, both $\boldsymbol{\alpha}$ subunits are likely to contribute towards airway inflammation. There appears to be some redundancy between HIF-1 $\boldsymbol{\alpha}$ and HIF-2 $\boldsymbol{\alpha}$, since they exhibit different (although overlapping) spectra of target genes [35]. Deleting each $\alpha$ subunit individually is likely to provide limited insight into the role of HIF-1 in the development of allergic airway inflammation. Furthermore, interpretation of data from these mice could be further confounded by the compensatory upregulation of one $\boldsymbol{\alpha}$ subunit that can occur when the other is decreased or ablated [36-39]. Hence, our ARNT conditional knock out mice are well suited for this study compared to individually knocked out HIF-1 $\boldsymbol{\alpha}$ or HIF- $2 \boldsymbol{\alpha}$ mice. It should be noted that although ARNT is also an obligatory subunit for the Aryl hydrocarbon receptor (AhR), C57BL/6 Ahr-null mice do not exhibit a reduced allergic lung inflammatory response after an OVA challenge protocol very similar to the one we performed here [40].
Using our conditionally knocked out mice, we observed a significant decrease in HIF-1 $\boldsymbol{\alpha}$ expression and more importantly a decrease in CCL2 expression; this observation was associated with a decreased inflammatory response (Figure 2C). This finding clearly demonstrates that CCL2 is under the regulation of HIF$\boldsymbol{\alpha}$ and that it is implicated in the allergic inflammatory response. Our findings provide evidence for the important involvement of HIF- $1 \boldsymbol{\alpha}$ in the regulation of CCL2 expression in allergic inflammatory disease. Altogether, the present study suggests that this is also likely to be the case in humans, with possible significant clinical implications.

We observed significant upregulation of HIF-1 $\boldsymbol{\alpha}$ and CCL2 in epithelial cells from bronchial lavage and lung tissue of asthmatic patients after exposure to allergen. Although one previous study correlated HIF- $1 \boldsymbol{\alpha}$ and $2 \boldsymbol{\alpha}$ overexpresion in bronchial lavage and lung tissue from asthmatic patients as compared to healthy controls, the levels of protein were only measured in pre-challenge conditions [19]. A recent study by our group demonstrates a significant increase of HIF-1 and its target gene VEGF after allergen challenge [21]. However, to our knowledge this is the first time that a direct correlation between HIF-1 $\boldsymbol{\alpha}$ and CCL2 expression has been shown in asthmatic patients after cat allergen challenge.

In the current study, we provide compelling evidence for a direct association between the expression of HIF-1 and CCL2 during allergic lung inflammation in the mouse using a combination of pharmacological and genetic approaches. We also provide evidence for this association in asthmatic patients. Our animal studies and supporting human data indicate an important role for the HIF-1 $\alpha$-mediated allergic inflammatory response possibly via regulation of the pro-inflammatory chemokine CCL2. Further studies will have to address the direct regulation of CCL2 by HIF-1 during allergic airway inflammation. Furthermore, although CCL2 may not be considered an ideal pharmacotherapeutic target for the treatment of asthma, our results indicate that HIF- $1 \alpha$ may be used as a specific target.

\section{Competing interests}

The authors declare that they have no competing interests. Funding has been received from the Collaborative Research Grant from the University of California Institute for Mexico and the United States (UC MEXUS-CONACYT) (S.H-Y and O.H). Mexico Federal Funds Grant HIM/2008/034 (S.H.-Y., G.B.-G.), National Institutes of Health grants R01 CA28868 (O.H.), R01 HL080343 (E.K. and M.Z.), NIH/NHLBI R01-HL-080343 (D.T) and NIH/NCATS UL1-TR-000124 (E.K., M.Z and D.T).

\section{Authors contributions}

GJBG carried out the experimental models and immunostaining and performed the statistical analysis. IGB prepared the manuscript. MZ designed the human studies, and analyzed and interpreted the data. RHP designed the experimental models. MIV edited the manuscript. EGZ participated in carrying out the experimental models. GAA carried out the

immunofluorescence assay. BB edited the manuscript. MR participated in the 
design of the human study and provided samples. EK designed the human studies and analyzed and interpreted the data. DPT participated in the design of the human study. $\mathrm{OH}$ participated in the design of the $\mathrm{KO}$ mouse study and edited the manuscript. SHY conceived the study, and participated in its design and coordination, and prepared the manuscript.

All authors read and approved the final manuscript.

\section{Author details}

${ }^{1}$ Hospital Infantil de Mexico, Federico Gomez, Unidad de Investigacion en Enfermedades Oncologicas, Dr. Marquez No 262, Col. Doctores, Delegacion Cuahutemos, Mexico City, Mexico CP. 06720. ${ }^{2}$ Facultad de Medicina Programa de Postgrado; Doctorado en Ciencias Biomedicas UNAM, Mexico City, Mexico. ${ }^{3}$ Department of Pathology and Laboratory Medicine, David Geffen School of Medicine at UCLA, Los Angeles, CA, USA. ${ }^{4}$ ChemRisk, LLC, Aliso Viejo, CA, USA. ${ }^{5}$ Department of Clinical Immunology and Allergy, David Geffen School of Medicine, UCLA, Los Angeles, CA, USA. ${ }^{6}$ Experimental Pathology Section, Department of Pathology, National Institute of Medical Science and Nutrition, Salvador Zubiran, Mexico City, Mexico. ${ }^{7}$ Unidad de Investigacion Medica en Enfermedades Oncologicas, CMN SXXI, IMSS, Mexico City, Mexico. ${ }^{8}$ Departamento de Inmunología, Instituto de Investigaciones Biomédicas, UNAM, México, Mexico. ${ }^{9}$ Department of Medicine, David Geffen School of Medicine, UCLA, Los Angeles, CA, USA. ${ }^{10}$ Department of Microbiology Immunology and Molecular Genetics, UCLA, Los Angeles, CA, USA. ${ }^{11}$ Pulmonary and Critical Care Medicine, David Geffen School of Medicine, UCLA, Los Angeles, CA, USA.

Received: 22 February 2012 Accepted: 3 July 2012

Published: 23 July 2012

\section{References}

1. Bergeron C, Boulet LP: Structural changes in airway diseases: characteristics, mechanisms, consequences, and pharmacologic modulation. Chest 2006, 129(4):1068-1087.

2. Barnes PJ: Th2 cytokines and asthma: an introduction. Respir Res 2001, 2(2):64-65.

3. Chung KF, Barnes PJ: Cytokines in asthma. Thorax 1999, 54(9):825-857.

4. Gould HJ, Sutton BJ: IgE in allergy and asthma today. Nat Rev Immunol 2008, 8(3):205-217

5. Rollins BJ: Monocyte chemoattractant protein 1: a potential regulator of monocyte recruitment in inflammatory disease. Mol Med Today 1996, 2(5):198-204.

6. Sica A, Wang JM, Colotta F, Dejana E, Mantovani A, Oppenheim JJ, Larsen CG, Zachariae CO, Matsushima K: Monocyte chemotactic and activating factor gene expression induced in endothelial cells by IL-1 and tumor necrosis factor. J Immunol 1990, 144(8):3034-3038.

7. Taub DD, Proost P, Murphy WJ, Anver M, Longo DL, van DJ, Oppenheim JJ: Monocyte chemotactic protein-1 (MCP-1), -2 , and -3 are chemotactic for human T lymphocytes. J Clin Invest 1995, 95(3):1370-1376.

8. Campbell EM, Charo IF, Kunkel SL, Strieter RM, Boring L, Gosling J, Lukacs NW: Monocyte chemoattractant protein-1 mediates cockroach allergeninduced bronchial hyperreactivity in normal but not CCR2-/- mice: the role of mast cells. J Immunol 1999, 163(4):2160-2167.

9. Karpus WJ, Lukacs NW, Kennedy KJ, Smith WS, Hurst SD, Barrett TA: Differential CC chemokine-induced enhancement of T helper cell cytokine production. J Immunol 1997, 158(9):4129-4136.

10. Lukacs NW, Chensue SW, Karpus WJ, Lincoln P, Keefer C, Strieter RM, Kunkel SL: C-C chemokines differentially alter interleukin-4 production from lymphocytes. Am J Pathol 1997, 150(5):1861-1868.

11. Sousa AR, Lane SJ, Nakhosteen JA, Yoshimura T, Lee TH, Poston RN: Increased expression of the monocyte chemoattractant protein-1 in bronchial tissue from asthmatic subjects. Am J Respir Cell Mol Biol 1994 10(2):142-147

12. Holgate ST, Bodey KS, Janezic A, Frew AJ, Kaplan AP, Teran LM: Release of RANTES, MIP-1 alpha, and MCP-1 into asthmatic airways following endobronchial allergen challenge. Am J Respir Crit Care Med 1997, 156 (5):1377-1383.

13. Gonzalo JA, Lloyd CM, Wen D, Albar JP, Wells TN, Proudfoot A, Martinez A, Dorf M, Bjerke T, Coyle AJ, Gutierrez-Ramos JC: The coordinated action of CC chemokines in the lung orchestrates allergic inflammation and airway hyperresponsiveness. J Exp Med 1998, 188(1):157-167.
14. Mojsilovic-Petrovic J, Callaghan D, Cui H, Dean C, Stanimirovic DB, Zhang W: Hypoxia-inducible factor-1 (HIF-1) is involved in the regulation of hypoxiastimulated expression of monocyte chemoattractant protein-1 (MCP-1/ CCL2) and MCP-5 (CCl12) in astrocytes. J Neuroinflammation 2007, 4:12.

15. Lando D, Peet DJ, Whelan DA, Gorman JJ, Whitelaw ML: Asparagine hydroxylation of the HIF transactivation domain a hypoxic switch. Science 2002, 295(5556):858-861.

16. Lando D, Peet DJ, Gorman JJ, Whelan DA, Whitelaw ML, Bruick RK: FIH-1 is an asparaginyl hydroxylase enzyme that regulates the transcriptional activity of hypoxia-inducible factor. Genes Dev 2002, 16(12):1466-1471

17. Vincent KA, Feron O, Kelly RA: Harnessing the response to tissue hypoxia: HIF-1 alpha and therapeutic angiogenesis. Trends Cardiovasc Med 2002, 12(8):362-367.

18. Dehne N, Brune B: HIF-1 in the inflammatory microenvironment. Exp Cell Res 2009, 315(11):1791-1797.

19. Lee SY, Kwon S, Kim KH, Moon HS, Song JS, Park SH, Kim YK: Expression of vascular endothelial growth factor and hypoxia-inducible factor in the airway of asthmatic patients. Ann Allergy Asthma Immunol 2006, 97(6):794-799

20. Huerta-Yepez S, Baay-Guzman GJ, Garcia-Zepeda R, Hernandez-Pando R, Vega MI, Gonzalez-Bonilla C, Bonavida B: 2-Methoxyestradiol (2-ME) reduces the airway inflammation and remodeling in an experimental mouse model. Clin Immunol 2008, 129(2):313-324.

21. Huerta-Yepez S, Baay-Guzman GJ, Bebenek IG, Hernandez-Pando R, Vega MI, Chi L, Riedl M, Diaz-Sanchez D, Kleerup E, Tashkin DP, Gonzalez FJ, Bonavida $B$, Zeidler M, Hankinson O: Hypoxia inducible factor promotes murine allergic airway inflammation and is increased in asthma and rhinitis. Allergy 2011, 66(7):909-918.

22. Tomita S, Sinal CJ, Yim SH, Gonzalez FJ: Conditional disruption of the aryl hydrocarbon receptor nuclear translocator (Arnt) gene leads to loss of target gene induction by the aryl hydrocarbon receptor and hypoxiainducible factor 1alpha. Mol Endocrinol 2000, 14(10):1674-1681

23. Takagi S, Tojo H, Tomita S, Sano S, Itami S, Hara M, Inoue S, Horie K, Kondoh G, Hosokawa K, Gonzalez FJ, Takeda J: Alteration of the 4-sphingenine scaffolds of ceramides in keratinocyte-specific Arnt-deficient mice affects skin barrier function. J Clin Invest 2003, 112(9):1372-1382.

24. Corren J, Spector S, Fuller L, Minkwitz M, Mezzanotte W: Effects of zafirlukast upon clinical, physiologic, and inflammatory responses to natural cat allergen exposure. Ann Allergy Asthma Immunol 2001, 87(3):211-217

25. Zeidler MR, Goldin JG, Kleerup EC, Kim HJ, Truong DA, Gjertson DW, Kennedy NJ, Newman KB, Tashkin DP, Silverman JM, Corren J: Small airways response to naturalistic cat allergen exposure in subjects with asthma. J Allergy Clin Immunol 2006, 118(5):1075-1081.

26. Majamaa K, Gunzler V, Hanauske-Abel HM, Myllyla R, Kivirikko Kl: Partia identity of the 2-oxoglutarate and ascorbate binding sites of prolyl 4-hydroxylase. J Biol Chem 1986, 261(17):7819-7823.

27. Kozak KR, Abbott B, Hankinson O: ARNT-deficient mice and placental differentiation. Dev Biol 1997, 191(2):297-305.

28. Maltepe E, Schmidt JV, Baunoch D, Bradfield CA, Simon MC: Abnormal angiogenesis and responses to glucose and oxygen deprivation in mice lacking the protein ARNT. Nature 1997, 386(6623):403-407.

29. Steinke JW, Woodard CR, Borish L: Role of hypoxia in inflammatory upper airway disease. Curr Opin Allergy Clin Immunol 2008, 8(1):16-20.

30. Lee KS, Kim SR, Park HS, Park SJ, Min KH, Lee KY, Choe YH, Hong SH, Han HJ, Lee YR, Kim JS, Atlas D, Lee YC: A novel thiol compound, $\mathrm{N}$-acetylcysteine amide, attenuates allergic airway disease by regulating activation of NF-kappaB and hypoxia-inducible factor-1alpha. Exp Mol Med 2007, 39(6):756-768.

31. Lee KS, Kim SR, Park SJ, Park HS, Min KH, Jin SM, Lee MK, Kim UH, Lee YC: Peroxisome proliferator activated receptor-gamma modulates reactive oxygen species generation and activation of nuclear factor-kappaB and hypoxia-inducible factor 1alpha in allergic airway disease of mice. J Allergy Clin Immunol 2006, 118(1):120-127.

32. Guo J, Lu W, Shimoda LA, Semenza GL, Georas SN: Enhanced interferongamma gene expression in T Cells and reduced ovalbumin-dependent lung eosinophilia in hypoxia-inducible factor-1-alpha-deficient mice. Int Arch Allergy Immunol 2009, 149(2):98-102.

33. Warnecke C, Griethe W, Weidemann A, Jurgensen JS, Willam C, Bachmann S, Ivashchenko Y, Wagner I, Frei U, Wiesener M, Eckardt KU: Activation of 
the hypoxia-inducible factor-pathway and stimulation of angiogenesis by application of prolyl hydroxylase inhibitors. FASEB J 2003, 17(9): 1186-1188.

34. Kallio PJ, Pongratz I, Gradin K, McGuire J, Poellinger L: Activation of hypoxia-inducible factor 1alpha: posttranscriptional regulation and conformational change by recruitment of the Arnt transcription factor. Proc Natl Acad Sci U S A 1997, 94(11):5667-5672.

35. Hu CJ, Wang LY, Chodosh LA, Keith B, Simon MC: Differential roles of hypoxia-inducible factor 1alpha (HIF-1alpha) and HIF-2alpha in hypoxic gene regulation. Mol Cell Biol 2003, 23(24):9361-9374.

36. Carroll VA, Ashcroft M: Role of hypoxia-inducible factor (HIF)-1alpha versus HIF-2alpha in the regulation of HIF target genes in response to hypoxia, insulin-like growth factor-l, or loss of von Hippel-Lindau function: implications for targeting the HIF pathway. Cancer Res 2006, 66(12):6264-6270.

37. Raval RR, Lau KW, Tran MG, Sowter HM, Mandriota SJ, Li JL, Pugh CW, Maxwell PH, Harris AL, Ratcliffe PJ: Contrasting properties of hypoxiainducible factor 1 (HIF-1) and HIF-2 in von Hippel-Lindau-associated renal cell carcinoma. Mol Cell Biol 2005, 25(13):5675-5686.

38. Wang V, Davis DA, Haque M, Huang LE, Yarchoan R: Differential gene upregulation by hypoxia-inducible factor-1alpha and hypoxia-inducible factor-2alpha in HEK293T cells. Cancer Res 2005, 65(8):3299-3306.

39. Warnecke C, Zaborowska Z, Kurreck J, Erdmann VA, Frei U, Wiesener M, Eckardt KU: Differentiating the functional role of hypoxia-inducible factor (HIF)-1alpha and HIF-2alpha (EPAS-1) by the use of RNA interference: erythropoietin is a HIF-2alpha target gene in Hep3B and Kelly cells. FASEB J 2004, 18(12):1462-1464.

40. Lawrence BP, Denison MS, Novak H, Vorderstrasse BA, Harrer N, Neruda W, Reichel C, Woisetschlager M: Activation of the aryl hydrocarbon receptor is essential for mediating the anti-inflammatory effects of a novel lowmolecular-weight compound. Blood 2008, 112(4):1158-1165.

doi:10.1186/1465-9921-13-60

Cite this article as: Baay-Guzman et al:: HIF-1 expression is associated with CCL2 chemokine expression in airway inflammatory cells: implications in allergic airway inflammation. Respiratory Research 2012 13:60

\section{Submit your next manuscript to BioMed Central and take full advantage of:}

- Convenient online submission

- Thorough peer review

- No space constraints or color figure charges

- Immediate publication on acceptance

- Inclusion in PubMed, CAS, Scopus and Google Scholar

- Research which is freely available for redistribution 